\title{
On the Bounds of Separability in Sensor Networks
}

\author{
B Santhana Krishnan \\ Electrical Engineering Department, Indian Institute of Technology, Bombay \\ Powai, Mumbai, India - 400076. \\ Email: skrishna@ee.iitb.ac.in
}

\begin{abstract}
A pair of target locations are separable if sensor observations can distinguish between the following choices: no targets are present, one target is present at either of the locations or a target is present at each location. The sensors of interest in this paper are binary proximity sensors, whose binary outputs are functions of the distance between the sensor and target. Sensors are deployed randomly according to a Poisson distribution. The probability that two target locations at a distance of $r$ between them are separable is derived. This is extended to derive the probability of having at least $Z$ among $M$ uniformly distributed target locations to be non-separable from the origin. The bounds on this probability are expressed as a function of the sensor density $\lambda$.
\end{abstract}

\section{INTRODUCTION}

A sensor network consists of a number of sensor nodes which monitor an activity (e.g. target tracking) or a condition (e.g. temperature) in a certain sensing area $\mathscr{A}$ of interest. Localisation algorithms in sensor network applications typically determine the location of targets using the sensor locations and observations. The output of the localisation algorithm for a particular target is an area within which the target is present. This area is called the uncertainty region. The uncertainty region area is a measure of localisation performance.

This paper's focus, the separability problem, is different from standard localisation problems. If $t_{0}, t_{1}$ are two target locations, the objective is to determine which of these two locations if any contains a target. Separability, which is formally defined in Section II, is an attribute of a pair of target locations. If target locations $t_{0}$ and $t_{1}$ are separable, then the sensor observations can correctly decode any of the following scenarios: (1) two targets are present(one each at both the locations) (2) one target is present(at any one of the locations) (3) no targets are present. Separability is a function of the distance $r$ between $t_{0}$ and $t_{1}$.

The interest of this paper is in sensor networks that detect specific events. Assume that a set of candidate target locations are available in $\mathscr{A}$. Consider a reference location in $\mathscr{A}$ and assume the reference location to be the origin. The objective is to characterise the number of targets present by estimating bounds on the tail distribution of the number of targets nonseparable from the origin.

Assume that the sensors are deployed according to a spatial Poisson process and the sensors used are proximity sensors. The output of a proximity sensor is a function of the distance between the sensor and targets in its neighbourhood. The vector of sensor outputs is collectively used to estimate the unknown, for example the target locations in a localisation problem.

The analytical bounds on performance of the separability algorithm are useful in answering practical design questions. Consider the following, if there are $M$ uniformly distributed targets in the sensing area and let $z: 0 \leq z \leq 1$ be a constant, what is the probability that there are at least $M z$ non-separable targets? The answer to this question is the main contribution of this paper. In particular, the bounds on the probability that there are at least $M z$ non-separable targets as a function of the sensor density $\lambda$ have been derived.

\section{A. Previous work}

As mentioned earlier, this paper is related to localisation, but it is significantly different. See Patwari et. al [1] for a survey on localisation algorithms in the context of sensor networks. Mudumbai and Madhow [2] and Wang et. al. [3] derive two relevant results that are used to derive the bounds on the number of separable targets.

Mudumbai and Madhow [2] consider two possible target locations, say $t_{0}$ and $t_{1}$. It is known that exactly one target is present at either $t_{0}$ or $t_{1}$. Proximity sensors are deployed according to a homogeneous Poisson process. Let $N$ be the number of sensors. From a set of $N$ noisy observations a binary hypothesis testing problem of choosing between the two target locations is analysed. They evaluate the probability of error in detection as a function of the distance $r$ between $t_{0}$ and $t_{1}$ and derive information theoretic bounds on the error in detection.

Wang et. al. [3] define a minimum distance, $\tilde{r}$, such that the network can distinguish any pair of points if the distance between them is larger than $\tilde{r}$. An upper bound on the localisation error as a function of $\tilde{r}$ has been derived.

Our paper is an extension of Mudumbai and Madhow [2]. The extensions are: (1) the ability to choose between all four target availability configurations for a set of two target locations, defined as separability (2) count the number of targets that are not separable from a fixed target at the origin using separability and (3) obtain bounds on the number of non-separable targets.

\section{B. Notations and Preliminaries}

$\mathscr{A}$ is a finite sensing area which includes the origin. Sensors are distributed according to a homogeneous Poisson process of density $\lambda$. The number of sensors is a Poisson random variable denoted by $N$ with mean $\lambda|\mathscr{A}|$. Let $\left\{s_{j}\right\}, j=1,2, \ldots, N$ 
be the set of sensor locations. Poisson distribution implies independent increments and restricts the number of sensors in an infinitesimally small area to one, with high probability.

Let $\left\{t_{i}\right\}, i=1,2, \ldots, M$ be the set of $M$ possible target locations indexed by $i$. Let $x_{i}$ be an indicator variable such that $x_{i}=1$ if there is a target at $t_{i}$ and $x_{i}=0$ otherwise. The binary vector $\mathrm{x}$ of length $M$, termed target configuration vector, gives the locations where targets are actually present. Consider $\mathscr{A}$ to be sufficiently large. To avoid edge effects, consider the sensors and targets to be on the surface of a torus as in [4].

Let $d_{i j}$ denote the distance between target location $t_{i}$ and sensor location $s_{j}$, i.e. $d_{i j}=\left\|t_{i}-s_{j}\right\|_{2}$. The sensor outputs are conditionally independent if the sensor and target locations are known. There are two types of proximity sensors used in this paper.

1) Ideal Binary Proximity (IBP) Sensors give a Boolean output of 1 if there exists at least one target within the circle of radius $R_{s}$ centred at the sensor location and 0 otherwise. Singh et. al. [5] prove that, if the set of targets have all pairwise distances large enough such that no sensor can sense any two targets, then ideal binary sensing permits precise counting of targets.

2) A binary proximity (BP) sensor gives a Boolean output of 1 if it "senses" at least one target. If $x_{i}=1$, a sensor at $s_{j}$ senses the target at $t_{i}$ with probability $P_{j i}=f\left(d_{i j}\right), \quad f$ is a non-increasing function of the distance. Let $y_{j i}$ be the indicator variable that sensor $j$ senses target $i$. There are multiple targets in $\mathscr{A}$, the output $y_{j}$ is the Boolean OR operation of Bernoulli random variables $y_{j i}$, denoted as $\operatorname{Bern}\left(P_{j i}\right) x_{i}$.

$$
y_{j}=\vee_{i=1}^{M} y_{j i}=\vee_{i=1}^{M} \operatorname{Bern}\left(P_{j i}\right) x_{i}
$$

The derivation of the probability of having at least $z$ nonseparable target locations from the origin using IBP sensors, and the bounds on this probability are derived in the following section.

\section{SeParability FROM The ORIGIN USING IBP SENSORS}

Definition 1. Separability of two candidate locations $t_{0}, t_{1}$ is the ability to distinguish between all possible configuration vectors $\mathbf{x}=\left[x_{0}, x_{1}\right]$ defined for $\left(t_{0}, t_{1}\right)$.

Consider the origin to be a candidate target location $t_{0}$. Consider $t_{1}, t_{2}$ to be two additional target locations which are chosen according to a uniform distribution. In this section, using IBP sensors, the probability that $\left(t_{0}, t_{1}\right)$ are not separable is derived first. Then the probability that $t_{1}$ and $t_{2}$ are both not separable from $t_{0}$ is derived. Using these probabilities, the expectation and variance of the number of targets not separable from the origin and the bounds on the number of such targets have been derived.

Consider the target location $t_{1}$ at a distance of $r$ from $t_{0}$. The homogeneity of sensor distribution allows us to ignore the orientation of $t_{1}$ with respect to $t_{0}$. For IBP sensors, Fig. 1 shows four disjoint areas marked
$A_{0}(r), A_{1}(r), A_{0,1}(r), A^{-}(r)$. An IBP sensor in either $A_{0}(r)$ or $A_{1}(r)$ only detects the respective target $t_{0}$ or $t_{1}$. The output of a sensor in $A_{0,1}(r)$ distinguishes between $\mathbf{x}=[0,0]$ and the other configurations. The output of a sensor in $A^{-}(r)$ cannot be used in making a decision about locations $t_{0}, t_{1}$.

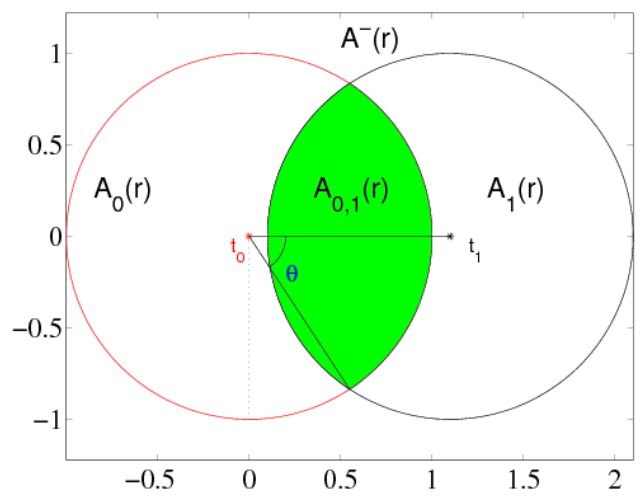

Fig. 1. Separability of $t_{1}$ from $t_{0}$.

\section{A. Probability of Separability}

The probability of $t_{0}$ and $t_{1}$ being separable is the probability that there are sensors in both $A_{0}(r)$ and $A_{1}(r)$; see Fig. 1. Since $\left|A_{0}(r)\right|=\left|A_{1}(r)\right|$, the subscript can be ignored. The probability of having zero sensors in the area $A(r)$ is calculated using (2)

$$
\begin{aligned}
A(r) & =R_{s}^{2}(\pi-[2 \theta-\sin (2 \theta)]) \\
\text { where } \theta & =\arccos \left(\frac{r}{2 R_{s}}\right) .
\end{aligned}
$$

Let $\psi\left(t_{1}\right)$ be the indicator variable corresponding to target location $t_{1}$ defined in (3)

$$
\psi\left(t_{1}\right):= \begin{cases}1 & \text { if locations }\left(t_{0}, t_{1}\right) \text { are non-separable } \\ 0 & \text { otherwise. }\end{cases}
$$

The conditional probability of $t_{1}$ and $t_{0}$ being non-separable is given by

$$
\begin{aligned}
\operatorname{Pr}\left(\psi\left(t_{1}\right)=1 \mid r\right) & =1-\operatorname{Pr}\left(N_{A(r)}>0\right) \operatorname{Pr}\left(N_{A(r)}>0\right) \\
& =e^{-\lambda A(r)}\left[2-e^{-\lambda A(r)}\right] .
\end{aligned}
$$

The targets are distributed such that their distances from $t_{0}$ follow the density function $f_{R}(r)$. The probability that a randomly picked candidate target location $t_{1}$ is non-separable from $t_{0}$, is calculated by unconditioning (4) with respect to $r$; see (5). While unconditioning over target realisations, targets that are at a distance farther than $2 R_{s}$, from $t_{0}$, are separable if they are both covered since no sensor commonly senses two such targets. Let $\rho=\operatorname{Pr}\left(\psi\left(t_{1}\right)=1\right)$. $\rho$ is an exponentially decreasing function of $\lambda$ from the expressions.

$$
\begin{aligned}
& \operatorname{Pr}\left(\psi\left(t_{1}\right)=1 \mid r>2 R_{s}\right)=1-\left(1-e^{-\lambda \pi R_{s}^{2}}\right)^{2} \\
& \rho=\int_{r} f_{R}(r) \operatorname{Pr}\left(\psi\left(t_{1}\right)=1 \mid r\right) d r
\end{aligned}
$$

$\rho$ is the probability that a target at a random location in $\mathscr{A}$ is non-separable from $t_{0}$. 


\section{B. Joint Probability of two candidate locations being non- separable from $t_{0}$}

Consider two possible locations $t_{1}, t_{2}$ distributed uniformly in $\mathscr{A}$. Let the polar coordinates of the locations be $t_{1}=$ $\left(r_{1}, \theta_{1}\right), t_{2}=\left(r_{2}, \theta_{2}\right)$. The events $\left(t_{0}, t_{1}\right)$ being non-separable and $\left(t_{0}, t_{2}\right)$ being non-separable are not independent. To characterise the separability probability i.e. the probability that two target locations $t_{1}, t_{2}$ are jointly non-separable from $t_{0}, \operatorname{Pr}\left(\psi\left(t_{1}\right)=1\right.$ and $\left.\psi\left(t_{2}\right)=1 \mid r_{1}, \theta_{1}, r_{2}, \theta_{2}\right)$, is calculated using the following steps:

1) Reduction of coordinates: By using the symmetry of correlation, rotation invariance, without loss of generality the polar coordinates are assumed to be $t_{1} \triangleq$ $\left(r_{1}, 0\right), t_{2} \triangleq\left(r_{2}, \theta\right) . r_{1}, r_{2}$ are distributed according to $f_{R}(r), \theta$ is distributed according to $f_{\Theta}(\theta)$.

2) Define area $A_{0}$ such that, a sensor in $A_{0}$ detects the target at $t_{0}$. Similarly, if there is a sensor in the area $\tilde{A}_{0}$, it would only detect the target at $t_{0}$; see Fig. 2. Areas $\tilde{A}_{01}, \tilde{A}_{12}$ and $\tilde{A}_{012}$ are similarly defined. The individual area calculations are available in [6].

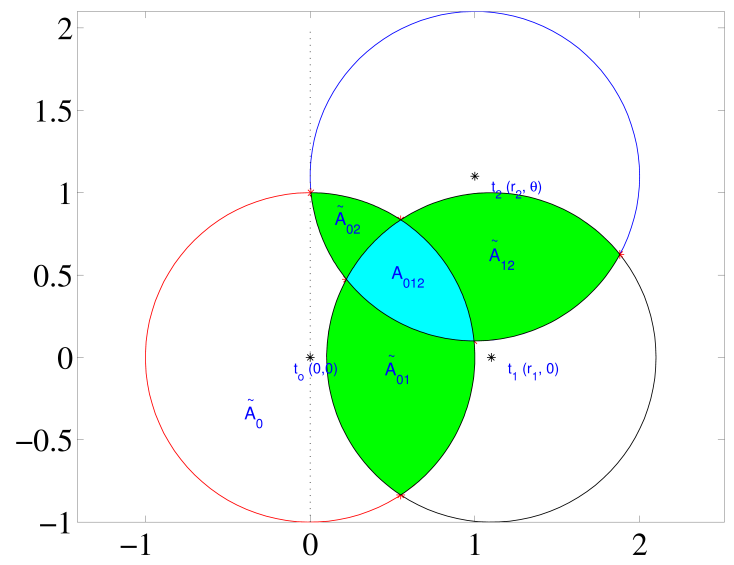

Fig. 2. Joint Separability of two target locations from $t_{0}$

Let $\phi_{12}$ be the indicator variable which corresponds to the two locations $t_{1}, t_{2}$ being jointly non-separable from $t_{0}$

$\phi_{12}:= \begin{cases}1 & \text { if }\left(t_{0}, t_{1}\right) \text { and }\left(t_{0}, t_{2}\right) \text { are both non-separable } \\ 0 & \text { otherwise. }\end{cases}$

$$
\begin{aligned}
& P\left(\phi_{12}=1 \mid r_{1}, r_{2}, \theta\right)= \\
& \quad 1-P\left(N_{\tilde{A}_{0}}>0 \& N_{\tilde{A}_{1}}>0 \& N_{\tilde{A}_{2}}>0\right)
\end{aligned}
$$

The areas in the above expression are non-overlapping, the individual probabilities are calculated similar to (4). $\phi_{12}=1$ implies, all the 8 configurations of $\left\{t_{0}, t_{1}, t_{2}\right\}$ are decodable.

Let $\Psi_{12}$ be the indicator variable that corresponds to $\left(t_{1}, t_{0}\right)$ being separable assuming $t_{2}$ is absent and $\left(t_{2}, t_{0}\right)$ being separable assuming $t_{1}$ is absent, a weaker condition than the joint separability. $\left(t_{1}, t_{0}\right)$ are separable in any one of the four disjoint configurations:

1) Sensors are present in $\tilde{A}_{0}$ and $\tilde{A}_{12}$.
2) Sensors are present in $\tilde{A}_{0}$ and $\tilde{A}_{1}$, while no sensors are present in $\tilde{A}_{12}$.

3) No sensors are present in $\tilde{A}_{0}$ and sensors are present in $\tilde{A}_{02}$ and $\tilde{A}_{12}$.

4) No sensors are present in both $\tilde{A}_{0}$ and $\tilde{A}_{12}$ sensors are present in $\tilde{A}_{02}$ and $\tilde{A}_{1}$.

The condition required for $\left(t_{2}, t_{0}\right)$ to be separable in each of the above configuration is: for 1) no extra condition required, for 2) sensors should be present in $\tilde{A}_{2}$, for 3 ) sensors should be present in $\tilde{A}_{01}$ and for 4 ) sensors should be present in $\tilde{A}_{01}$ and in $\tilde{A}_{2}$. Let $\sigma^{2}$ denote the unconditional probability of $\Psi_{12}$.

$$
\begin{aligned}
& P\left(\Psi_{12}\right.\left.=0 \mid r_{1}, r_{2}, \theta\right)=P\left(N_{\tilde{A}_{0}}>0 \& N_{\tilde{A}_{12}}>0\right) \\
&+P\left(N_{\tilde{A}_{0}}>0 \& N_{\tilde{A}_{12}}=0 ; \& N_{\tilde{A}_{1}}>0\right) P\left(N_{\tilde{A}_{2}>0}\right) \\
&+P\left(N_{\tilde{A}_{0}}=0 \& N_{\tilde{A}_{02}}>0 \& N_{\tilde{A}_{12}}>0\right) P\left(N_{\tilde{A}_{01}>0}\right) \\
&+P\left(N_{\tilde{A}_{0}}=0 \& N_{\tilde{A}_{02}}>0 \& N_{\tilde{A}_{12}}=0 \& N_{\tilde{A}_{1}}>0\right) \\
& \sigma^{2}= \int_{r_{1}} \int_{\theta} \int_{r_{2}} P\left(N_{\tilde{A}_{01}>0} \& N_{\tilde{A}_{2}}>0\right) . \\
& d\left(F_{R}\left(r_{2} \mid r_{1}\right)\right) d\left(F_{\Theta}(\theta)\right) d\left(F_{R}\left(r_{1}\right)\right) .
\end{aligned}
$$

\section{EXTENDING TO SEPARABILITy OF $M$ TARGET LOCATIONS}

Let $M$ be the number of possible target locations and $Z(M)$ to be the fraction of the number of target locations that are not separable from $t_{0}$. It is necessary to note that $\psi\left(t_{i}\right)$ are not independent. In calculating $\mathrm{E}[Z(M)], \operatorname{Var}[Z(M)]$, for $\psi\left(t_{i}\right)$ it is assumed that all the other $(M-1)$ targets at $t_{k}, k \in$ $\{1,2 \ldots i-1, i+1, \ldots M\}$ are absent.

$$
\begin{aligned}
Z(M) & :=\frac{1}{M} \sum_{i=1}^{M} \psi\left(t_{i}\right) \\
\mathrm{E}[Z(M)] & =\frac{\mathrm{E}\left[\sum_{i=1}^{M} \psi\left(t_{i}\right)\right]}{M}=\rho .
\end{aligned}
$$

Variance of $Z(M)$ is derived using the covariance formula as

$$
\begin{aligned}
\operatorname{Var}(Z(M)) & =\frac{1}{M^{2}} \sum_{i=1}^{M} \sum_{j=1}^{M} \operatorname{COV}\left[\psi\left(t_{i}\right) \psi\left(t_{j}\right)\right] \\
\operatorname{COV}\left[\psi\left(t_{i}\right) \psi\left(t_{j}\right)\right] & =\mathrm{E}_{t_{i}, t_{j}}\left[\Psi_{i j}-\psi\left(t_{i}\right) * \psi\left(t_{j}\right)\right]=\sigma^{2}-\rho^{2} \\
\operatorname{Var}(Z(M)) & =\left(\frac{M-1}{M}\right)\left(\sigma^{2}-\rho^{2}\right)+\frac{\rho-\rho^{2}}{M} \approx \sigma^{2}-\rho^{2} .
\end{aligned}
$$

The network is 'translation invariant', i.e. any of the $(M+1)$ target locations could have been considered to be the origin, and the mean and variance of the number of non-separable targets would have been identical. Translation invariance is owing to the homogeneous sensor distribution and uniform target distribution. $\mathrm{E}[Z(M)], \operatorname{Var}(Z(M))$ are functions of $\rho, \sigma$ which are in turn functions of $\lambda, R_{s}$ and $M$.

Averaging over the number of target locations: If the number of candidate locations is also random, the expected number of targets non-separable from $t_{0}$ can be estimated. Hence the mean and variance of $Z$ will be that of $Z(M)$ averaged over the number of target locations $M$. 


\section{A. Chebyshev Bounds}

The one-sided Chebyshev inequality is used to derive the bounds on the number of non-separable targets, let $g \in \mathbb{R}, g>$ 0 be a constant. The bounds on having at least $z$ fraction of non-separable target locations is calculated as

$$
\operatorname{Pr}(Z(M) \geq z) \leq \frac{\operatorname{Var}(Z(M))}{\operatorname{Var}(Z(M))+(z-\mathrm{E}[Z(M)])^{2}} \cdot \forall z \geq \rho
$$

The upper bound is valid for $z \geq \rho$. The lower bound can be derived for the random variable $V(M)=1-Z(M)$, using $g \in \mathbb{R}, g>0$, a constant. The lower bound is valid for $z \leq \rho$.

$$
\operatorname{Pr}(Z(M) \geq z)>1-\frac{\operatorname{Var}(Z(M))}{\operatorname{Var}(Z(M))+(z-\mathrm{E}[Z(M)])^{2}} \forall z \leq \rho
$$

\section{B. Chernoff Bounds}

An approximation to the Chernoff bound ${ }^{1}$ (given below) is derived.

$$
\operatorname{Pr}(Z(M) \geq z) \leq \min _{\theta>0} e^{-\theta z} \mathrm{E}\left[e^{\theta Z(M)}\right] .
$$

Upper Bound on $\operatorname{Pr}(Z(M) \geq z)$

The term $\mathrm{E}\left[e^{\theta Z(M)}\right]$, in the above expression, cannot be simplified further since, $\psi\left(t_{i}\right), \psi\left(t_{j}\right), i \neq j$ are not independent. The Jensen's inequality separates the terms of $\mathrm{E}\left[e^{\theta Z(M)}\right]$. The terms break out into individual identical expectations, since expectation is linear. Using Jensen's inequality [7]:

$$
\begin{aligned}
e^{\theta Z(M)}=e^{\frac{\theta}{M} \sum_{i=1}^{M} \psi\left(t_{i}\right)} & \leq \sum_{i=1}^{M} \frac{e^{\theta \psi\left(t_{i}\right)}}{M} \\
\operatorname{Pr}(Z(M) \geq z) & \leq \min _{\theta>0} \sum_{i=1}^{M} e^{-\theta z} \mathrm{E}\left[\frac{e^{\theta \psi\left(t_{i}\right)}}{M}\right] \\
& =\min _{\theta>0}\left(1-\rho+\rho e^{\theta}\right) e^{-\theta z} . \\
\text { at the minimum } e^{\theta} & =\frac{1-\rho}{\rho} \frac{z}{1-z} ; \theta>0 \Rightarrow z>\rho \\
\forall z>\rho \quad \operatorname{Pr}(Z(M) \geq z) & \leq\left(\frac{\rho}{z}\right)^{z}\left(\frac{1-\rho}{1-z}\right)^{1-z} .
\end{aligned}
$$

The upper bound is trivial if $z=\rho$. If the number of target locations $M \rightarrow \infty$, the bounds obtained considering $\psi\left(t_{i}\right)$ to be independent can still be used to obtain meaningful bounds[8].

Lower Bound on $\operatorname{Pr}(Z(M) \geq z)$

The lower bound calculation is similar to the upper bound.

The bound on the variable $V(M)=1-Z(M)$ is calculated.

$$
\begin{aligned}
\operatorname{Pr}(V(M) \geq 1-z) & \leq \min _{\theta>0} e^{-\theta(1-z)} E\left[e^{\theta V(M)}\right] \\
\operatorname{Pr}(Z(M) \leq z) & \leq \min _{\theta>0} e^{\theta z}\left(1-\rho+\rho e^{-\theta}\right)
\end{aligned}
$$$$
\forall z \leq \rho \operatorname{Pr}\left(Z(M) \geq z+\frac{1}{M}\right) \geq 1-\left(\frac{\rho}{z}\right)^{z}\left(\frac{1-\rho}{1-z}\right)^{1-z}
$$

${ }^{1}$ The variable $\theta$ used in the Chernoff bound is different from the one used to denote angle in Fig. 1.

\section{Results AND Discussion}

Consider $R_{S}=1$ and $\mathscr{A}$ is a square of side $10 . \rho$ for different values of $\lambda$ have been evaluated in Fig 3 . As mentioned earlier, it is seen that $\rho(\lambda)$ is an exponentially decreasing function.

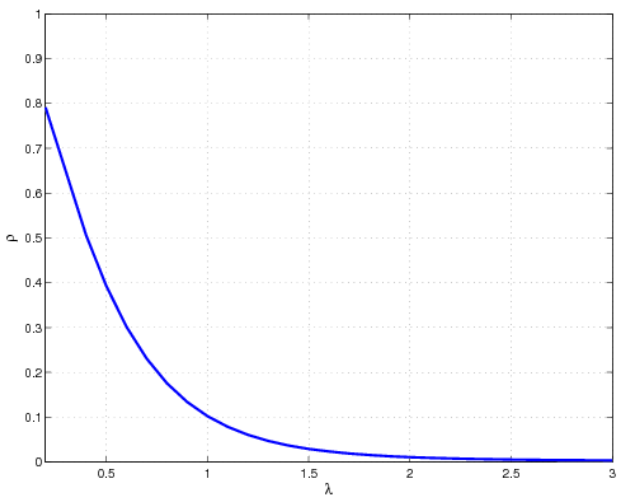

Fig. 3. Numerical evaluation of $\rho$ as a function of $\lambda$.

The upper and lower bounds of $\operatorname{Pr}(Z \geq z)$, for $R_{s}=$ $1, M=25, \lambda=0.8 \mathscr{A}$ is square of side 10 , is shown in Fig. 4. From (9), (10), (11), (12) and Fig. 4 both the Chernoff bound obtained using Jensen's inequality and the Chebyshev bound are trivial at $z=\rho$.

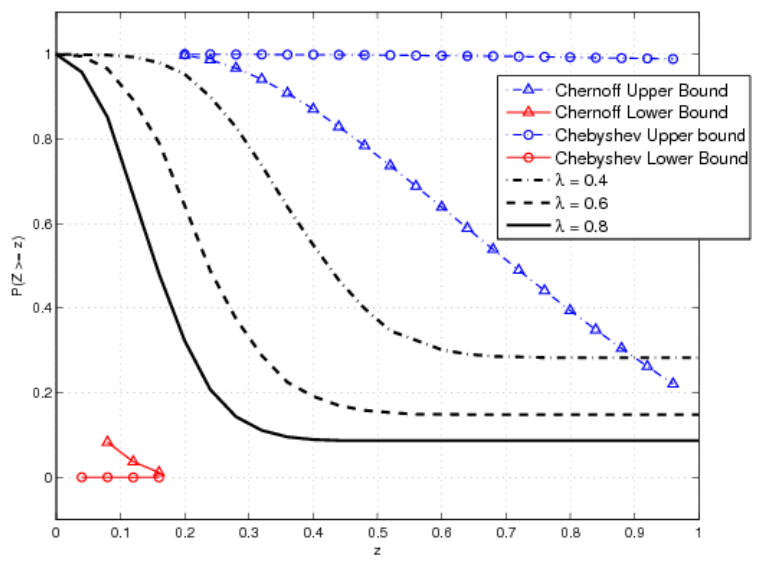

Fig. 4. Empirical probabilities of $M z$ targets non-separable from the origin

For $\lambda \in\{0.4,0.6,0.8\}$, the empirical tail distribution of having more than $M z$ targets non-separable from the origin is shown in Fig 4.

This set of results can be extended to BP sensors. The difference is that for the IBP sensors $\psi\left(t_{i}\right)$ is a deterministic binary value, while for the BP case, it would be a probability. Using a maximum a-posteriori (MAP) based detection rule, following [2], bounds on the separability performance of two targets for a specific sensing function $f_{1}\left(d_{i j}\right)$ can 2) be obtained. Similarly obtaining bounds for more general distributions (other than the Poisson) and for a scaling analysis, $\lim _{M \gg 1, R_{s} \rightarrow 0} \operatorname{Pr}(Z \geq z)$, would give better insights into bounds in practical deployments. 


\section{ACKNOWLEDGEMENT}

The author would like to thank his advisors Prof. D Manjunath and Prof. Bikash K Dey for introducing the problem, and for all the discussions and corrections to this paper.

\section{REFERENCES}

[1] Neal Patwari et. al., "Locating the nodes: cooperative localization in wireless sensor networks," IEEE Signal Processing Magazine, vol. 22, no. 4, pp. 54-69, July 2005.

[2] Raghuraman Mudumbai and Upamanyu Madhow, "Information theoretic bounds for sensor network localization," in Proc. IEEE Intl. Symp. on Inform. Theory (ISIT), July 2008, pp. 1602-1606.

[3] Wei Wang, Vikram Srinivasan, Bang Wang, and Kee-Chaing Chua, "Coverage for target localization in wireless sensor networks," in Proc. Intl. Conference on Information Processing in Sensor Networks (IPSN), 2006, pp. 118-125.

[4] Peter Hall, Introduction to the Theory of Coverage Processes, John Wiley and Sons, New York, 1988.

[5] Jaspreet Singh, Upamanyu Madhow, Rajesh Kumar, Subhash Suri, and Richard Cagley, "Tracking multiple targets using binary proximity sensors," in Proc. Intl. Conference on Information Processing in Sensor Networks (IPSN), 2007, pp. 529-538.

[6] B. Santhana Krishnan, "Localisation in sensor networks and information theory, http://www.ee.iitb.ac.in/student/ $\sim$ skrishna/apsreports/," Tech. Rep., Annual Progress Seminar -1, Electrical Engineering Dept, Indian Institute of Technology, Bombay, 2009.

[7] Thomas M. Cover and Joy A. Thomas, Elements of information theory, Wiley-Interscience, New York, NY, USA, 1991.

[8] Alan Siegel, "Toward a usable theory of chernoff bounds for heterogeneous and partially dependent random variables," http://cs.nyu.edu/faculty/siegel/HHf.pdf, 1992. 